\title{
Nouvelle version du chapitre «Rapports» dans le TARMED à compter du 1er avril 2006
}

Hanspeter Kuhn, avocat;

Lucia Rabia, avocate;

Dania Ischi, licenciée en droit; Simon Stettler, avocat, licencié en droit
Correspondance: Service juridique de la FMH Elfenstrasse 18 CH-3000 Berne 15 lex@fmh.ch

\section{Résumé}

Il est possible d'appliquer, dans le respect de la loi, la disposition contractuelle sur les rapports figurant dans le TARMED révisé. Pour ce faire, il s'agit notamment de suivre la notice (ou «feuillet thématique») que le PFPD a publiée à ce sujet en 2002 et de procéder par étapes. Avant de demander un rapport médical (ou des informations tirées d'un tel rapport), l'assureur ou son médecinconseil doit poser des questions. A cet effet, il indique au médecin ou à l'hôpital la raison pour laquelle il a besoin d'informations en précisant la thématique qu'il doit examiner et les questions concrètes qui s'y rapportent:

- évaluation du droit à des prestations, économicité; concrètement:...

- coordination avec l'assureur LAA concerné; concrètement:...

- coordination avec l'office AI concerné; concrètement:...

- coordination avec l'office AM concerné; concrètement:...

- recours contre un tiers responsable; concrètement:...

- incapacité de travail couverte par une assurance d'indemnités journalières selon la LAMal; concrètement:...

Le médecin traitant décide s'il va répondre directement et à qui (médecin-conseil ou administration de la caisse-maladie), ou prendre tout d'abord contact avec le patient.

\section{La position révisée IC-00.06-1 soulève des questions}

La nouvelle version du TARMED approuvée par le Conseil fédéral et valable à dater du $1^{\text {er }}$ avril 2006 contient le texte suivant sous IC-00.06-1: «Tous les rapports doivent être remis à l'assureur ou au médecin-conseil de l'assureur, s'il les demande; dans ce cas, les dispositions de la protection des données sont applicables.» [1] Des médecins nous ont demandé comment procéder face à cette nouvelle version rédactionnelle. Nous sommes d'avis que cette disposition contractuelle sur les rapports, telle qu'elle figure dans le TARMED révisé, peut être appliquée dans le respect de la loi. A cet égard, il est déterminant que tous les acteurs de la santé s'entendent sur la signification concrète des expressions «s'il les demande» et «dans ce cas, les dispositions de la protection des données sont applicables». En limitant pour l'instant nos commentaires à la LAMal, nous estimons que les aspects suivants sont importants pour agir correctement:

\section{La convention TARMED accorde aux parties contractantes une marge de ma- nœuvre importante, mais pas illimitée}

Eugster et Luginbühl définissent la liberté d'action dont bénéficient les parties contractantes de la façon suivante:

(traduction FMH) «Dans ce contexte [il s'agit de l'art. 42 LAMal; remarque des auteurs], le législateur accorde aux parties contractantes une marge de manœuvre relativement grande mais pas illimitée. Les partenaires tarifaires ne doivent pas seulement respecter les dispositions contraignantes de la LAMal et les principes généraux relevant du droit législatif, administratif ou de l'assurance sociale, mais aussi tenir compte des principes centraux de la protection des données. [...] La plus haute importance est notamment accordée au principe de la proportionnalité (à savoir au critère du caractère indispensable). La réglementation tarifaire ne doit pas interférer de manière disproportionnée avec le droit d'autodétermination des assurés en matière d'information, protégé par l'art. 13 de la Constitution fédérale.» [2]

\section{Recherche d'information par l'assureur: de manière échelonnée, conformément au PFPD, aussi pour les rapports médicaux}

En 2002, le Préposé fédéral à la protection des données (PFPD) a rédigé une notice (ou «feuillet thématique») concernant les rapports opératoires et les lettres de sortie d'hôpital. Cette notice stipule à bon droit qu'il faut agir de manière échelonnée. Ce mode de faire est indiqué pour tous les rapports médicaux, donc aussi, par exemple, pour les rapports concernant un envoi ou un transfert à l'hôpital. Dans son rapport sur le TARMED du 22 juin 2004, le PFPD a fixé cet échelonnement comme principe général de la LAMal (et donc pas seulement pour les rapports d'opération et de sortie): «Dans les alinéas 3 et 4 de l'art. 42 LAMal, le législateur prévoit une communication échelonnée par le fournisseur de prestations des données relatives au traitement.» [3]

A notre sens, la phrase figurant dans le texte révisé du TARMED «dans ce cas, les dispositions de la protection des données sont applicables» signifie avant tout que les assurances, médecins et 
hôpitaux doivent agir de manière échelonnée. Voici un extrait de la notice du PFPD de 2002:

«1 ${ }^{\text {er }}$ degré: les hôpitaux et les homes établissent une facture détaillée et compréhensible.

$2^{\text {ème }}$ degré: si dans un cas d'espèce, l'assureur a besoin d'indications supplémentaires, il a la possibilité de poser par écrit à l'exécutant de la prestation des questions spécifiques et limitées au cas concret. Il lui incombe de motiver la nécessité de ces questions supplémentaires. Une copie de cette demande est envoyée à la personne assurée à titre d'information.

$3^{\text {ème }}$ degré: si ces informations supplémentaires ne suffisent exceptionnellement pas, l'assureur peut demander une lettre de sortie ou un rapport opératoire à l'intention de son médecin-conseil. Il doit motiver par écrit la nécessité de cette requête. Une copie en est envoyée à la personne assurée à titre d'information.»

\section{La LAMal n'utilise pas le terme de «rapport»; elle parle toujours des infor- mations nécessaires à I'assureur, peu importe où elles se trouvent}

La LAMal et les ordonnances y relatives ne mentionnent nulle part que les rapports écrits à l'attention d'autres médecins doivent aussi parvenir à l'assureur-maladie. Et ce à juste titre, puisque cette loi ne se soucie pas de ces rapports, mais des informations nécessaires à l'assureur ou à son

\section{Comment procéder lorsque la caisse-maladie demande des renseigne- ments ou un rapport médical? L'essentiel pour le lecteur pressé}

1. Que dois-je faire en ma qualité de médecin traitant?

Vous devez développer un sixième sens pour savoir dans quel cas il est opportun de discuter avec votre patient avant de transmettre des informations médicales à la caisse-maladie. II conviendra particulièrement de vous entretenir avec lui lorsqu'il

- $s^{\prime}$ agit de questions socialement délicates (affections psychiques, maladies sexuellement transmissibles, cancer), ou lorsque

- vous savez que votre patient se trouve dans une phase difficile (p. ex. changement d'emploi, divorce, candidature à une fonction politique).

2. Que puis-je faire en ma qualité de médecin?

La directive du Préposé fédéral à la protection des données (PFPD) est claire: vous avez le droit d'exiger que la caisse-maladie justifie sa demande d'information et envoie une copie de sa lettre au patient.

3. Quand est-ce que je risque, en tant que médecin, une condamnation pour cause de violation du secret médical (art. 321 du code pénal)?

- Lorsque vous répondez directement à une question de la caisse-maladie,

- que le patient se sent pris au dépourvu et dépose plainte et

- que le juge parvient à la conclusion que vous auriez dû, en l'espèce, compter avec le fait que le patient paierait lui-même la facture pour éviter que l'information en question ne parvienne à la caisse. médecin-conseil (peu importe l'endroit où ces informations sont saisies: dans un rapport, dans le dossier médical, ou seulement dans la tête du médecin). En vertu de l'art. 84 LAMal, l'assureur a le droit d'obtenir et de traiter les informations «qui lui sont nécessaires» pour accomplir sa tâche. Le PFPD précise dans sa notice: «Les assureurs ne peuvent recueillir que les données des assurés qui leur sont indispensables à remplir leurs tâches légales (principe de finalité).»

Et plus bas:

«Les lettres de sorties comme les rapports opératoires contiennent beaucoup d'informations sensibles. Les informations relatives aux assurés sont soit directement reconnaissables (p.ex. les diagnostics), soit exploitables de manière indirecte. Il est par conséquent possible de faire ressortir de ces documents d'autres informations qui concernent les maladies ou l'état psychique du patient, lesquelles informations ne sont pourtant pas liées à son hospitalisation ou à son séjour dans un home.

Les assureurs peuvent traiter ces données parce qu'elles leur sont indispensables à déterminer s'ils sont obligés de fournir une prestation. Les hôpitaux et les homes sont par conséquent autorisés à communiquer ces données aux assureurs.

Toutefois, une facture détaillée est en général suffisante pour déterminer l'obligation de l'assureur. Dans des cas particuliers, d'autres indications peuvent par contre se révéler nécessaires.»

\section{Les interventions techniques sont susceptibles d'être contrôlées mais la relation et les entretiens entre patient et médecin doivent être protégés}

Il est certes vrai que lorsque l'assureur doit prendre en charge les coûts de traitement, il obtient un grand nombre d'informations sur celui-ci et ses causes par le biais des positions tarifaires et par celui des médicaments et analyses facturés.

Par contre, il n'est pas et n'a jamais été dans l'intention du législateur que l'assureur obtienne le dossier médical dans son intégralité, car le patient perdrait alors toute confiance en la médecine qui deviendrait impossible à pratiquer. Le contenu des entretiens et la relation patient-médecin doivent, en particulier, rester confidentiels. Le secret professionnel (art. 321 du code pénal [4]) est défini de la même manière pour les médecins, les avocats et les ecclésiastiques. A titre de comparaison, même l'avocat commis d'office - et payé par l'Etat! - ainsi que l'ecclésiastique n'ont pas le droit de communiquer au juge compétent pour le paiement ou au trésorier de la paroisse ce que l'accusé ou le fidèle leur a confié, uniquement pour que la justice ou l'église puisse contrôler le temps consacré à la défense ou à la cure d'âmes. Dans ces trois cas, il en va du contenu central du secret professionnel [5].

L'assureur n'est jamais en droit de tout savoir. De ce fait, il n'y a pas lieu de lui envoyer le dos- 
sier médical complet (ou à son médecin-conseil) puisqu'il n'a jamais besoin de l'avoir en entier: ce n'est pas lui qui traite le patient. (Dans des cas rares et extrêmes, p.ex. lors de fort soupçon d'escroquerie, la compétence de s'occuper du dossier revient de toute façon au juge d'instruction et non plus à la caisse!)

\section{Le médecin traitant doit trier les informations et se demander ce dont a besoin I'assureur et dans quel but}

L'assureur ne doit pas tout savoir. Mais, conformément à la LAMal, il lui incombe d'obtenir les informations dont il a besoin pour ses investigations. Par conséquent, le médecin traitant doit effectuer un tri quand il reçoit des demandes de renseignements, et sélectionner les informations que seul le médecin-conseil doit recevoir et celles qui peuvent parvenir à l'administration de la caisse. La FMH et la Société des médecins-conseils sont d'accord sur ce point [6]. A cet effet, le médecin doit savoir dans quel but l'assureur a besoin des informations demandées. «Dans ce cas, les dispositions de la protection des données sont applicables» veut donc dire qu'avec sa demande, l'assureur communique au médecin ou à l'hôpital pour quelles raisons il a besoin d'informations.

Nous estimons qu'il incombe au collaborateur de l'assurance-maladie de noter les points obscurs et d'en discuter avec le service du médecin-conseil. S'il reste ensuite des questions non résolues, le médecin-conseil [7] s'adresse alors au médecin ou à l'hôpital en indiquant la thématique (voir ci-après) et les questions concrètes y afférentes. S'agit-il:

- d'une appréciation du droit à des prestations, d'une évaluation de l'économicité? [8] Pour le contrôle de l'économicité, l'indication médicale de même que les données sur l'examen ou le traitement effectué sont déterminantes. Les informations qui n'ont rien à voir dans ce contexte ne doivent pas être envoyées à l'assureur, peu importe qu'elles se trouvent dans le dossier médical manuscrit, dans des rapports établis par d'autres médecins ou ailleurs [9].

- de coordination avec l'assureur LAA? [10] On entend par là les questions du genre: la notion d'accident est-elle applicable? S'agit-il d'une maladie professionnelle, d'une lésion corporelle assimilée à un accident, ou des séquelles tardives d'un accident? [11]

- de coordination avec l'AI? On entend dans ce cas par exemple la question de savoir si les mesures prises sont appropriées pour une réinsertion professionnelle [12].

- de coordination avec l'AM? Ici interviennent des questions concernant le moment où la maladie est apparue ou le lien de causalité [13].
- d'un recours contre un tiers responsable? [14] Dans un tel cas, des indications relatives à la causalité de l'accident ou aux coûts futurs de traitement peuvent aussi jouer un rôle important.

- de questions concernant l'incapacité de travail dans le cadre d'une assurance d'indemnités journalières selon la LAMal? [15]

Le patient a le droit de choisir - le médecin doit donc savoir ce que veut le patient Conformément à la LAMal, le patient peut demander que les informations médicales soient transmises uniquement au médecin-conseil (art. $42,5^{\mathrm{e}}$ al. LAMal [16]). En outre, le patient peut toujours, en guise d'«ultime frein d'urgence», renoncer sans autre formalité aux prestations de la caisse s'il tient à protéger sa sphère privée.

En raison de ce droit de choisir réservé au patient, il revient au médecin traitant (et non pas à l'assureur ou à son médecin-conseil) de décider - s'il peut et a le droit de répondre directement à une demande de renseignements de la caisse ou

- s'il doit auparavant s'entretenir à ce sujet avec le patient.

Que peut-il se passer lorsque le médecin a envoyé à la caisse le rapport demandé sans en discuter avec son patient et que celui-ci dépose plainte pour violation du secret médical (secret professionnel selon l'art. 321 du code pénal)? Le médecin concerné peut envisager l'argument suivant pour sa défense: «Dans un arrêt daté du 9 octobre 2001, le TFA a décidé que la caisse a le droit de définir quelles informations sont nécessaires pour elle.» Néanmoins, et nous ne sommes pas les seuls de cet avis, cet arrêt du TFA est généralement considéré comme indéfendable [17]. De plus, le juge pénal n'est pas lié à l'appréciation d'une question effectuée par le juge de l'assurance sociale. De ce fait, il n'est pas possible de prévoir à l'avance comment il va prononcer son arrêt. Le risque d'une condamnation est particulièrement élevé lorsque le juge pénal estime que le patient, s'il avait été interrogé à temps sur sa décision, aurait renoncé au versement des prestations de la caisse-maladie pour éviter la transmission des informations en question à la caisse ou à son médecin-conseil.

\section{Références}

1 www.fmh.ch/ww/fr/pub/dienstleistungen/tarife/ tarmed/tarmed_browser.htm.

2 Eugster G, Luginbühl R. Datenschutz in der obligatorischen Krankenpflegeversicherung. In: Hürlimann B, Jacobs R, Poledna T (Hrsg.). Datenschutz im Gesundheitswesen. Zürich: Schulthess; 2001. S. 101ff. 
3 www.edsb.ch - Thèmes: Santé - TARMED et protection des données, Rapport du PFPD.

4 Art. 321 du Code pénal suisse

1. Les ecclésiastiques, avocats, défenseurs en justice, notaires, contrôleurs astreints au secret professionnel en vertu du code des obligations, médecins, dentistes, pharmaciens, sages-femmes, ainsi que leurs auxiliaires, qui auront révélé un secret à eux confié en vertu de leur profession ou dont ils avaient eu connaissance dans l'exercice de celle-ci, seront, sur plainte, punis de l'emprisonnement ou de l'amende. Seront punis de la même peine les étudiants qui auront révélé un secret dont ils avaient eu connaissance à l'occasion de leurs études. La révélation demeure punissable alors même que le détenteur du secret n'exerce plus sa profession ou qu'il a achevé ses études.

2. La révélation ne sera pas punissable si elle a été faite avec le consentement de l'intéressé ou si, sur la proposition du détenteur du secret, l'autorité supérieure ou l'autorité de surveillance l'a autorisée par écrit.

3. Demeurent réservées les dispositions de la législation fédérale et cantonale statuant une obligation de renseigner une autorité ou de témoigner en justice.

5 Günther Stratenwerth, spécialiste en droit pénal, a dégagé l'essentiel de cette disposition: «Les personnes exerçant les professions mentionnées ici ne peuvent accomplir leurs tâches de manière optimale que dans la mesure où leurs clients leurs font confiance sans réserve.» Stratenwerth G. Schweizerisches Strafrecht. Besonderer Teil II. Berne: Stämpfli; 2000. p. 381.

6 Pour les auteurs, la formulation «les informations que seul le médecin-conseil doit recevoir et celles qui peuvent parvenir à l'administration de la caisse» signifie aussi qu'il existe des informations concernant le traitement qui ne doivent parvenir ni au médecin-conseil ni à l'administration de la caisse - à savoir celles qui sont sans importance pour les questions posées par la caisse.

7 L'art. 5, $2^{\mathrm{e}}$ al., de la Convention relative aux médecins-conseils, Fonction du médecin-conseil, précise ce qui suit: «Des renseignements ne peuvent être fournis au nom du médecin-conseil que par lui-même ou par l'un des ses auxiliaires.»

8 LAMal, art. 84, $1^{\text {er }}$ al., let. a; LAMal, art. 32ss et 56

9 En particulier, l'assurance obligatoire des soins ne peut pas réduire le montant des prestations lors d'un comportement fautif du patient. (Il en va autrement dans la LAA: si l'assuré a commis une faute grave, les coûts de traitement sont entièrement pris en charge mais les indemnités journalières et les rentes peuvent être réduites).

10 LAMal $84,1^{\mathrm{er}}$ al. let. b (est aussi valable pour la coordination avec l'AI et l'AM).

11 Notion d'accident: art. 4 LPGA; maladies professionnelles: art. 9 LAA; lésions corporelles assimilées à un accident: art. 9 OLAA; rechutes et séquelles tardives: art. 11 OLAA.

12 Art. 8 LAI.

13 LAM, art. 5 (affection pendant le service) ou art. 6 (responsabilité en cas d'affection après le service: «l'assurance militaire répond de l'affection seulement s'il est établi au degré de vraisemblance pré pondérante que l'affection a été causée ou aggravée pendant le service ou seulement s'il est établi au degré de vraisemblance prépondérante qu'il s'agit de séquelles tardives ou de rechute d'une affection assurée.»

14 LAMal, art. 84, $1^{\mathrm{er}}$ al., let. e.

15 La LAMal continue de prévoir, à partir de l'art. 67 une assurance d'indemnités journalières facultative, mais malgré tout sociale, à laquelle s'appliquent les dispositions générales de la LAMal et pour laquelle le médecin-conseil exerce, notamment, la même fonction de consultant et de filtre que dans l'assurance obligatoire des soins. Le TFA l'a clairement établi dans son arrêt du 10 août 2004 (K 121/03), Assurance d'indemnités journalières selon la LAMal (traduction FMH): «Les assureurs-maladie doivent veiller, dans le cadre de la loi et des statuts, à ne verser que les prestations auxquelles l'assuré a véritablement droit; de ce fait, ils ont le droit et, le cas échéant, le devoir de vérifier les données de l'assuré et aussi celles du médecin (ATF 107 V 103 avec remarques; RAMA 1987 n K 738 p. 253). Selon la LAMal, la fonction de surveillance et de contrôle incombe aux médecins-conseils des assureurs-maladie dont la tâche a été élargie par rapport au droit en vigueur jusqu'à présent (art. $57,4^{\mathrm{e}}$ al., $2^{\mathrm{e}}$ phrase LAMal; ATF 127 V 47 s. considérant 2d). [...] En conséquence, le règlement des indemnités journalières de l'intimée, qui est déclaré applicable au ch. 6 du contrat collectif 2 , prévoit aussi expressément au ch. 8.1.3 que le membre doit, sur demande, se laisser examiner par un deuxième médecin ou par le médecin-conseil de la caisse.»

16 «Le fournisseur de prestations est fondé lorsque les circonstances l'exigent, ou astreint dans tous les cas, si l'assuré le demande, à ne fournir les indications d'ordre médical qu'au médecin-conseil de l'assureur, conformément à l'article 57.»

17 Le 13 octobre 2003, le tribunal arbitral du canton de Zurich a appliqué cet arrêt du TFA de 2001 tout en exprimant de vives critiques à l'égard du tribunal suprême: «So sehr dem Beklagten in Bezug auf diese - auch dem aufgehobenen Beschluss des Schiedsgerichts vom 25. Januar 2001 zugrunde gelegenen - Überlegungen zuzustimmen und so deutlich im vorliegenden Fall erkennbar ist, dass die Klägerin zur Überprüfung der Wirtschaftlichkeit der gemäss KVG vergüteten Leistungen objektiv nicht sämtliche Behandlungsunterlagen benötigt, sondern allenfalls diejenigen, welche für die Beurteilung der Dauer der Spitalbedürftigkeit erforderlich sind (aus der KVG-Grundversicherung hat sie ja Tagespauschalen vergütet), so offenkundig also erscheint, dass die Klägerin Operationsbericht, Narkoseprotokoll und Rechnungsdetails (vgl. Urk. 2/2/3) nicht zur Überprüfung der Leistungspflicht nach KVG, sondern zur Überprüfung der Leistungspflicht aus der privaten Zusatzversicherung (welcher mehr als $90 \%$ des Gesamtrechnungsbetrags belastet wurden) und/oder zu einem anderen Zweck anfordert, steht einer Einschränkung des klägerischen Akteneinsichtsrechts die dem Rückweisungsentscheid des Eidgenössischen Versicherungsgerichts zugrundeliegende Rechtsauffassung entgegen, an welche das Schiedsgericht im Rahmen der erneuten Beurteilung dieses Falles gebunden ist.» 